\title{
Análise do Desenvolvimento das espécies Eucalyptus grandis e Pinus elliotti no cerrado do Triângulo Mineiro
}

\author{
André Luís Teixeira Fernandes ${ }^{1}$ \\ Thaíla de Mello Florêncio ${ }^{2}$ \\ Marianne Fidalgo de Faria ${ }^{2}$ \\ ${ }^{1}$ Professor Doutor da Universidade de Uberaba \\ Curso de Engenharia Ambiental \\ Av. Nenê Sabino, 1801 Bairro Universitário - CEP:38055-500 - Uberaba - MG, Brasil \\ andre.fernandes@uniube.br \\ ${ }^{2}$ Universidade de Uberaba \\ Graduandas do Curso de Engenharia Ambiental \\ Av. Nenê Sabino, 1801 Bairro Universitário - CEP:38055-500 - Uberaba - MG, Brasil \\ thailamello@gmail.com,mariannefidalgo@uniube.br
}

\begin{abstract}
It was assessed the development of both Eucalyptus grandis and Pinus elliotti forests with 4,8 years old, considering height, squared diameter, volume (m3 ha-1) and annual medium increment (m3 ha-1 year-1) submitted, for comparison, to parcels with and without irrigation and diversifing nutriment supply; $100 \%, 50 \%$ and $25 \%$ of the recommended application of fertilizer. The irrigated treatments presented the biggest value of squared diameter, height, volume and annual medium increment. The total application of the recommended fertilizer was not necessarily responsible for the biggest statistic value.
\end{abstract}

Palavras-chave: Madeira Comercial, Produção Florestal, Irrigação

\section{Introdução}

O uso do eucalipto como madeira serrada se potencializou, nos últimos anos, através da otimização tecnológica através de progressos industriais, permitindo a propagação de culturas desse espécime pelo país (SERPA et al, 2003). O Brasil é o segundo colocado no ranking dos países com maior cobertura florestal no mundo. As condições de solo e clima são vantajosas para a silvicultura (MATTOS, JUVENAL 2002).

De acordo com a Associação Brasileira dos Produtores de Florestas Plantadas (ABRAF), a área plantada de pinus e eucalipto equivaleu à 5.313.417 ha em 2006, sendo que o ano de 2007 apresentou crescimento de 3,4\% totalizando 5.560.203 ha plantados. Durante o período 2005-2007, o aumento da área plantada foi de 318.428 ha, havendo um decréscimo de 1,4\% de área plantada de pinus e um aumento de $10,1 \%$ da área plantada do eucalipto.

Embora existam polêmicas quanto ao uso indiscriminado ou exploratório da água em irrigações de culturas, este é um processo antigo, e se mostra útil para aumentar a produtividade das mesmas, como nos afirma Silva, Faria e Reis (2003). A irrigação por gotejamento, amplamente utilizada na fruticultura, apresenta bons resultados (GALETI, 1989). Todavia pouco se sabe da sua aplicação no setor de reflorestamento de espécies comerciais.

Determinar a viabilidade da aplicação desse método é essencial para o sucesso de um empreendimento, pois tal tecnologia exige investimentos consideráveis e está relacionado ao uso elevado de insumos, sendo essencial a análise econônica do sistema e dos seus componentes (SILVA; FARIA; REIS, 2003). 


\section{Metodologia}

O Experimento foi iniciado em outubro de 2003, conduzido no Campus Experimental da Universidade de Uberaba (Fazenda Escola), Uberaba/MG. Foram analisadas 2 espécies florestais aos 4,8 anos - com e sem irrigação em nível de parcela e em nível de subparcela, foi testada a adubação a $100 \%, 50 \%$ e $25 \%$ da dose recomendada; totalizando 4 tratamentos com 4 repetições e 1667 plantas/hectare. A fertirrigação foi realizada por gotejamento e aplicação convencional para os tratamentos sequeiros. Cada parcela apresenta dimensões de $60,0 \mathrm{x}$ 53,5m (15 linhas, 53,5m; individualizadas nos tratamentos irrigados por válvulas elétricas com solenóides ligados a painel controlador) e espaçamento $4,0 \times 1,5 \mathrm{~m}$. O delineamento experimental utilizado foi o inteiramente casualizado.

Conforme análise feita anteriormente ao plantio, a área requereu calagem de 2 toneladas por hectare de calcário dolomítico, em área total.

Inicialmente, a adubação de plantio incluiu sulfato de amônio, yoorin e cloreto de potássio(08-28-16), $250 \mathrm{~g} /$ planta, parceladas em 4 vezes - 6, 12, 18 e 24 meses após plantio.

Nos tratamentos irrigados, a adubação foi parcelada em 10 meses, e no caso dos tratamentos sequeiros, parcelados em 3 aplicações, sendo essas em março, outubro e dezembro, época das chuvas.

Os dados foram coletados em 2008, a partir da primeira árvore de plantio mensurável (zigue-zague). A média das áreas das parcelas foi $124,60 \mathrm{~m}^{2} \mathrm{em}$ formato ligeiramente retangular.

Em campo, utilizou-se fita métrica graduada em milímetros para a mensuração da circunferência das árvores a $1,30 \mathrm{~m}$ da base e posterior conversão para o diâmetro. Foi utilizado o hipsômetro Eletrônico Haglof para mensuração das alturas, trena para a mensuração da área das parcelas, coletores de dados com software próprio, fita diamétrica, suta graduada em mm, motosserra e aparelho GPS. Para o cálculo dos parâmetros dendrométricos, utilizou-se um microcomputador padrão PC/INTEL com o sistema InvMétodo (volumes), CubMétodo (cubagem) e pacote estatístico SPSS. O cálculo volumétrico exigiu a cubagem de 7 árvores irrigadas e 7 não-irrigadas de cada espécie. Os modelos Volumétricos e Hipsométricos foram ajustados através de rotinas desenvolvidas nos Softwares estatísticos SPSS e Statigraphycs. Os testes estatísticos para circunferência à altura do peito (CAP), diâmetro à altura do peito (DAP) e altura foram realizados com software ANOVA one WAY. Os testes estatísticos para Incremento Médio Anual (IMA) foram realizados com software Assistat 7.5 beta.

Para acompanhamento climático, dados referentes à temperatura, umidade relativa, radiação solar, evapotranspiração e precipitação por mês foram coletados no período de junho de 2008 a junho de 2009 na estação meteorológica da Fazenda Escola.

\section{Resultados e Discussão}

Os valores dos resultados estatísticos da biometria florestal do eucalipto estão apresentados na tabela 1 . O diâmetro quadrático (DG) do eucalipto irrigado e $50 \%$ da nutrição foi o maior dos tratamentos: $19,5 \mathrm{~cm}$. Os tratamentos irrigados com $100 \%$ e $25 \%$ não diferiram significativamente. Dentre os tratamentos de sequeiros, o maior e o menor valores de DG foram, respectivamente, os dos tratamentos com $25 \%$ e $50 \%$ de adubação $(16,8 \mathrm{~cm}$ e $14,8 \mathrm{~cm}$ ). O tratamento eucalipto irrigado e $100 \%$ adubado e o de sequeiro com $50 \%$ de adubação apresentaram, respectivamente, a maior e a menor alturas médias $(24,1 \mathrm{~cm}$ e 20,8 
m) e também apresentaram, respectivamente, o maior e o menor valores de incremento médio anual (I.M.A.): $77,6 \mathrm{~m}^{3} \cdot \mathrm{ha}^{-1} \cdot$ ano $^{-1}$ e $56,0 \mathrm{~m}^{3} \cdot \mathrm{ha}^{-1} \cdot$ ano $^{-1}$.

Tabela 1: Resultados estatísticos do eucalipto sob diferentes tratamentos - DG, Altura, Volume e I.M.A.

\begin{tabular}{|c|c|c|c|c|c|}
\hline \multirow[t]{2}{*}{ Tratam. } & \multirow[t]{2}{*}{ Estatística } & \multirow{2}{*}{$\begin{array}{c}\text { DG } \\
(\mathrm{cm})\end{array}$} & $\begin{array}{c}\text { Alturas } \\
(\mathrm{m})\end{array}$ & \multirow{2}{*}{$\begin{array}{l}\text { Volume } \\
\mathrm{m} 3 \mathrm{cc} / \mathrm{ha}\end{array}$} & \multirow{2}{*}{$\begin{array}{c}\text { I.M.A. } \\
\text { m3/ha/ano }\end{array}$} \\
\hline & & & Média & & \\
\hline \multirow[t]{3}{*}{ E-IR-A } & Médias: & 17,1 & 24,1 & 376,1 & 77,6 \\
\hline & C.V.\% & 5,3 & 7,1 & 12,7 & \\
\hline & I.C. $95 \%$ & 8,4 & 11,4 & 20,2 & \\
\hline \multirow[t]{3}{*}{ E-IR-B } & Médias: & 19,5 & 23,5 & 328,6 & 67,8 \\
\hline & C.V.\% & 15,5 & 7,2 & 22,5 & \\
\hline & I.C. $95 \%$ & 24,7 & 11,5 & 35,9 & \\
\hline \multirow[t]{3}{*}{ E-IR-C } & Médias: & 17,2 & 23,7 & 365,8 & 75,4 \\
\hline & C.V.\% & 6,2 & 8,2 & 9,5 & \\
\hline & I.C. $95 \%$ & 9,8 & 13,1 & 15,1 & \\
\hline \multirow[t]{3}{*}{ E-NI-A } & Médias: & 16,6 & 22,8 & 334,9 & 69,1 \\
\hline & C.V.\% & 5,1 & 8,3 & 16,4 & \\
\hline & I.C. $95 \%$ & 8,1 & 13,2 & 26,0 & \\
\hline \multirow[t]{3}{*}{ E-NI-B } & Médias: & 14,8 & 20,8 & 271,8 & 56,0 \\
\hline & C.V.\% & 13,0 & 20,3 & 33,5 & \\
\hline & I.C. $95 \%$ & 20,7 & 32,3 & 53,3 & \\
\hline \multirow[t]{3}{*}{ E-NI-C } & Médias: & 16,8 & 21,0 & 304,9 & 62,9 \\
\hline & C.V.\% & 11,4 & 14,5 & 20,1 & \\
\hline & I.C. $95 \%$ & 18,1 & 23,1 & 32,0 & \\
\hline
\end{tabular}

Os valores dos resultados estatísticos da biometria florestal do pinus estão apresentados na tabela 2. O tratamento de pinus irrigado e 100\% adubado apresentou o maior DG (16,9 $\mathrm{cm})$. Os tratamentos irrigados com $50 \%$ e $25 \%$ de adubação tiveram DG próximos $(15,1 \mathrm{e}$ $15,8 \mathrm{~cm}$ respectivamente). As espécies não-irrigadas não tiveram diferença significativa.

$\mathrm{O}$ tratamento de pinus com maior altura média (11,6 metros) foi o irrigado e $100 \%$ adubado. A menor altura média apresentada (10 metros) correspondeu ao tratamento sequeiro e $100 \%$ adubado. O maior I.M.A. correspondeu ao tratamento irrigado com $100 \%$ de adubação $\left(35,6 \mathrm{~m}^{3} \mathrm{ha}^{-1} \mathrm{ano}^{-1}\right)$. O tratamento com o menor valor de I.M.A. $\left(20,8 \mathrm{~m}^{3} \mathrm{ha}^{-1} \mathrm{ano}^{-1}\right)$ foi o sequeiro com $100 \%$ de adubação.

Tabela 2: Resultados estatísticos do pinus sob diferentes tratamentos - DG, Altura, Volume e I.M.A.

\begin{tabular}{|c|c|c|c|c|}
\hline Tratam. & Estatística & $\begin{array}{l}\mathrm{DG} \\
(\mathrm{cm}\end{array}$ & $\frac{\text { Alturas (m) }}{\text { Média }}$ & $\begin{array}{l}\text { Volume } \text { I.M.A. } \\
\text { m3cc/ha m3/ha/ano }\end{array}$ \\
\hline
\end{tabular}




\begin{tabular}{llcccc}
\hline P-IR-A & Médias: & 16,9 & 11,6 & 172,7 & 35,6 \\
& C.V.\% & 9,1 & 3,1 & 6,5 & \\
& I.C. 95\% & 14,5 & 5,0 & 10,4 & \\
\hline P-IR-B & Médias: & 15,1 & 10,6 & 153,4 & 31,6 \\
& C.V.\% & 20,2 & 19,4 & 50,5 & \\
& I.C. 95\% & 32,2 & 30,8 & 80,4 & \\
\hline \multirow{2}{*}{ P-IR-C } & Médias: & 15,8 & 10,9 & 143,1 & 29,5 \\
& C.V.\% & 14,4 & 17,2 & 44,3 & \\
& I.C. 95\% & 22,9 & 27,4 & 70,4 & \\
\hline \multirow{2}{*}{ P-NI-A } & Médias: & 14,3 & 10,0 & 101,0 & 20,8 \\
& C.V.\% & 8,3 & 11,7 & 30,9 & \\
& I.C. 95\% & 13,2 & 18,7 & 49,1 & \\
\hline \multirow{2}{*}{ P-NI-B } & Médias: & 14,8 & 10,2 & 113,1 & 23,3 \\
& C.V.\% & 8,0 & 12,1 & 17,0 & \\
& I.C. 95\% & 12,7 & 19,3 & 27,0 & \\
\hline P-NI-C & Médias: & 14,3 & 10,2 & 117,9 & 24,3 \\
& C.V.\% & 6,5 & 12,2 & 27,2 & \\
& I.C. 95\% & 10,4 & 19,4 & 43,3 & \\
\hline
\end{tabular}

O teste de Tukey revelou não haver diferença estatística com relação ao IMA tanto para o eucalipto como para o pinus a $5 \%$ de probabilidade sendo aceita a hipótese de nulidade.

Houve diferença estatística para valores de Altura do Eucalipto e de CAP, DAP e altura para o pinus.

O teste de normalidade de KS Lilliefors indicou que não houve normalidade de distribuição nos tratamentos das duas espécies.

Pelo teste de homocedasticidade de Bartlett, houve diferença para a altura da espécie de eucalipto e para CAP, DAP e altura do pinus.

Aplicou-se então o teste de Fisher, pormenorizando as variâncias dos tratamentos. Os tratamentos de eucalipto fertirrigados com $100 \%, 50 \%$ e $25 \%$ da dose recomendada correspondem aos números 1,2 e 3 . Os tratamentos sequeiros com $100 \%, 50 \%$ e $25 \%$ da dose recomendada, aos números 4,5 e 6 . Tratamentos não relacionados na tabela 14 não apresentaram diferença entre si.

Tabela 3: Teste de Fisher - Altura eucalipto

\begin{tabular}{cc}
\hline Tratamentos & $\mathrm{p}$ \\
\hline $1 \times 2$ & 0,0014 \\
$1 \times 6$ & 0,013 \\
$2 \times 1$ & 0,0014 \\
$2 \times 3$ & 0,00427 \\
$2 \times 4$ & 0,016 \\
$3 \times 2$ & 0,00427 \\
$4 \times 2$ & 0,016 \\
$6 \times 1$ & 0,013 \\
\hline
\end{tabular}


O teste de Fisher ainda foi aplicado para o pinus, explicitando as variâncias dos tratamentos para CAP (tabela 4), DAP (tabela 5) e altura (tabela 6). Assim como na análise do eucalipto, os tratamentos de pinus fertirrigados com $100 \%, 50 \%$ e $25 \%$ da dose recomendada correspondem aos números 1,2 e 3 . Os tratamentos sequeiros com $100 \%, 50 \%$ e $25 \%$ da dose recomendada, aos números 4,5 e 6 . Tratamentos não relacionados nas tabelas não apresentaram diferença entre si a $5 \%$ de probabilidade.

Tabela 4: Teste de Fisher - CAP pinus

\begin{tabular}{cc}
\hline Tratamentos & $\mathrm{p}$ \\
\hline $1 \times 4$ & 0,000668 \\
$1 \times 5$ & 0,014556 \\
$2 \times 4$ & 0,012477 \\
$4 \times 1$ & 0,000668 \\
$4 \times 2$ & 0,012477 \\
$4 \times 6$ & 0,028275 \\
$5 \times 1$ & 0,014556 \\
$6 \times 4$ & 0,028275 \\
\hline
\end{tabular}

Tabela 5: Teste de Fisher - DAP pinus

\begin{tabular}{cc}
\hline Tratamentos & $\mathrm{p}$ \\
\hline $1 \times 4$ & 0,000668 \\
$1 \times 5$ & 0,014558 \\
$2 \times 4$ & 0,012477 \\
$4 \times 1$ & 0,000668 \\
$4 \times 2$ & 0,012477 \\
$4 \times 6$ & 0,02827 \\
$5 \times 1$ & 0,014558 \\
$6 \times 4$ & 0,02827 \\
\hline
\end{tabular}

Tabela 6: Teste de Fisher - Altura pinus

\begin{tabular}{cc}
\hline Tratamentos & $\mathrm{p}$ \\
\hline $1 \times 4$ & 0,00207 \\
$1 \times 5$ & 0,021116 \\
$2 \times 4$ & 0,013984 \\
$4 \times 1$ & 0,00207 \\
$4 \times 2$ & 0,013984 \\
$4 \times 6$ & 0,010914 \\
$5 \times 1$ & 0,021116 \\
$6 \times 4$ & 0,010914 \\
\hline
\end{tabular}

A avaliação econômica mostrou que o pinus e o eucalipto possuem um potencial de fazer $\mathrm{R} \$ 1.025$ e $\mathrm{R} \$ 3.332$ por hectare ao ano. . Se o eucalipto for processado a carvão, cada hectare 
pode dar $416 \mathrm{mst} /$ há no ano 5 , ou $231 \mathrm{~m}^{3}$ de carvão. Desta forma, se o eucalipto for vendido em pé por $\mathrm{R} \$ 40 / \mathrm{mst}$ ele geraria uma renda em 5 anos de $\mathrm{R} \$ 16.640$ por hectare. Se for processado e vendido como carvão o potencial total da margem de lucro seria R\$ 120 por metro cúbico de carvão, ou R $\$ 27.720$. Considerando o valor presente de investimento em um hectare de eucalipto com alta tecnologia a $\mathrm{R} \$ 3.300$ por hectare, o retorno excede $\mathrm{o}$ investimento em 38\% numa margem total de lucro. Se o eucalipto for processado a carvão, o retorno do investimento em 5 anos é de 53\% numa margem total de lucros por ano.

\section{Conclusão}

Conforme esperado, os maiores valores referentes ao eucalipto para altura, volume e incremento médio anual foi apresentado pelo tratamento irrigado e adubado com o total da dose recomendada. O maior diâmetro quadrático foi o do eucalipto irrigado e tratado com $50 \%$ da dose recomendada de adubação.

Com relação ao pinus, o tratamento irrigado e com $100 \%$ da adubação recomendada apresentou os maiores valores dos índices estatísticos (diâmetro quadrático, altura, volume e incremento médio anual).

Quando adicionado fertilizantes sem a adição conjunta de água, os valores de altura e diâmetro para pinus não diferiram entre si, enquanto que os valores de incremento médio anual apresentaram diferença de até 3 metros cúbicos por hectare ao ano.

Os tratamentos de eucalipto não-irrigados com $100 \%$ e $25 \%$ de fertilizantes recomendados tiveram resultados próximos de I.M.A., altura média e diâmetro quadrático.

Apesar de haver uma resposta econômica positiva para a fertirrigação das florestas, o lucro não é considerável para uma floresta de 4,8 anos.

Os testes de análise de variância do CAP e DAP do eucalipto não revelaram diferença entre tratamentos, diferentemente da altura, havendo diferença estatística. Para o pinus, os testes de análise de variância acusaram diferenças estatísticas para CAP, DAP e altura. Os testes de análise de variância do I.M.A. não mostraram diferenças significativas entre os tratamentos de ambas as espécies. Da mesma maneira, nos testes de comparações de médias do I.M.A. foi aceita a hipótese de nulidade, não havendo diferença entre os tratamentos de ambas as espécies.

\section{Referências}

\section{ABRAF, Anuário estatístico da Associação Brasileira de Produtores de Florestas} Plantadas: ano base 2007. ABRAF, Brasília, 2008.

\section{ABRAF, Anuário estatístico da Associação Brasileira de Produtores de Florestas} Plantadas: ano base 2006. ABRAF, Brasília, 2007.

GALETI, P. A. Conservação do Solo; Reflorestamento - Clima. 2 edição. Campinas, Instituto Campineiro de Ensino Agrícola - Brasil, 1989. 233, 237 p.

MATTOS, Thais L; JUVENAL, René L. G.. O setor florestal no Brasil e a importância do reflorestamento. BNDES Setorial. Rio de Janeiro, nº 16, p 3-30, set 2002. 
SERPA, Pedro Nicolau et al . Avaliação de algumas propriedades da madeira de Eucalyptus grandis, Eucalyptus saligna e Pinus elliotti. Rev. Árvore, Viçosa, v. 27, n. 5, out. 2003 . Disponível em: $<$ http://www.scielo.br/scielo.php?script=sci_arttext\&pid=S010067622003000500015\&lng=pt\&nrm=iso $>$. Acesso em: 19 jan. 2009. doi: $10.1590 / \mathrm{S} 0100-67622003000500015$.

SILVA, Adriana L. da; FARIA, Manoel A. de; REIS, Ricardo P.. Viabilidade técnicoeconômica do uso do sistema de irrigação por gotejamento na cultura do cafeeiro. Rev. bras. eng. agríc. ambient., Campina Grande, v. 7, n. 1, 2003 . Disponível em: $<$ http://www.scielo.br/scielo.php?script=sci_arttext\&pid=S141543662003000100007\&lng=pt\&nrm=iso $>$. Acesso em: 08 Abr 2008. doi: $10.1590 / \mathrm{S} 1415-43662003000100007$ 